\title{
Existence results in Banach space for a nonlinear impulsive system
}

\author{
Hasib Khan ${ }^{1,2 *}$, Aziz Khan ${ }^{3 *}$, Thabet Abdeljawad ${ }^{4}$ and Abdulwasea Alkhazzan ${ }^{5}$
}

\section{"Correspondence:}

hasibkhan13@yahoo.com; azizkhan927@yahoo.com

${ }^{1}$ College of Engineering Mechanics

and Materials, Hohai University, Nanjing, P.R. China

${ }^{3}$ Department of Mathematics, University of Peshawar, Peshawar, Pakistan

Full list of author information is available at the end of the article

\begin{abstract}
We deal with three important aspects of a generalized impulsive fractional order differential equation (DE) involving a nonlinear $p$-Laplacian operator: the existence of a solution, the uniqueness and the Hyers-Ulam stability. Our problem involves Caputo's fractional derivative. For these goals, we establish an equivalent fractional integral form of the problem and use a topological degree approach for the existence and uniqueness of the solution (EUS). Next, we check the stability of the suggested problem and then demonstrate the results via an illustrative example. In the literature, we could not find articles on the Hyers-Ulam stability of the impulsive fractional order DEs with $\phi_{p}$ operator.
\end{abstract}

MSC: 34B82; 26A33; 45N05

Keywords: Impulsive fractional differential equations; Caputo's fractional derivative; Existence and uniqueness of positive solution; Hyers-Ulam stability

\section{Introduction}

Nowadays, fractional calculus has drawn interest of experts working in numerous applied fields. Mathematical models by using fractional order DEs have been considered in control theory, viscoelastic theory, biology, fluid dynamics, hydrodynamics, image processing, signals, and computer networking. For details, we suggest [1-8].

Scientists are exploring different scientific aspects of fractional calculus. The existence and uniqueness of solutions (EUS) are popular studies. For this purpose, scientists are using numerous scientific approaches. Fixed point theorems are useful techniques for this study. Here, we highlight some recent and useful scientific results of well-known scientists in the field. For example, Agarwal et al. [9] provided a detailed description of the impulsive fractional DEs using Lyapunov functions and overviewed results for the stability in Caputo's sense. Wang et al. [10] studied the existence of solutions and applications for different classes of impulsive fractional DEs from the available literature. Hu et al. [11] considered a nonlinear system of fractional order DEs with $p$-Laplacian at resonance. Jafari et al. [12] studied EUS in Banach space for boundary value problems (BVPs) of fractional order DEs with $p$-Laplacian. Khan et al. [13] proved EUS and the Hyers-Ulam stability for a class of BVPs based on fractional order DEs and nonlinear $p$-Laplacian operator. Baleanu et al. [14] considered a hybrid system of fractional order DEs with $p$-Laplacian operator for the analysis of EUS and they given illustrated their results with examples. Khan et al. [15] proved several types of stability theorems for a fractional order DE with $p$-Laplacian

(c) The Author(s) 2019. This article is distributed under the terms of the Creative Commons Attribution 4.0 International License (http://creativecommons.org/licenses/by/4.0/), which permits unrestricted use, distribution, and reproduction in any medium, provided you give appropriate credit to the original author(s) and the source, provide a link to the Creative Commons license, and indicate if changes were made. 
involving two types of fractional order derivatives, that is, Caputo and Riemann-Liouville types. Raheem and Maqbul [16] studied oscillatory behavior for an impulsive fractional order DE with the help of inequality technique. Wang and Zhang [17] established EUS for an impulsive class of fractional DEs with Hadamard fractional order derivatives and presented applications. Cong and Tuan [18] considered a fractional order DE with delay for the study of EUS.

Recently, some authors considered fractional DEs with $p$-Laplacian operator for the existence and stability results. Here, we highlight some related contributions of the scientists. For instance, Hu et al. [11] considered a nonlinear system of fractional order DEs with $p$ Laplacian at resonance. Jafari et al. [12] studied EUS in Banach space for boundary value problems (BVPs) of fractional order DEs with $p$-Laplacian. Khan et al. [13] proved EUS and Hyers-Ulam stability for a class of BVPs based on fractional order DEs and a nonlinear $p$-Laplacian operator. Baleanu et al. [14] considered a hybrid system of fractional order DEs with a $p$-Laplacian operator for the analysis of EUS and they have illustrated their results with examples. Khan et al. [15] proved several types of stability theorems for a fractional order DE involving a nonlinear $p$-Laplacian operator. Abbas et al. [19] considered a partial functional DE for the analysis of the Hyers-Ulam stability and existence results and provided two examples for applications of their results.

Recently, Liu et al. [20] studied the following impulsive fractional differential equation for the existence and uniqueness of solutions and also provided an application:

$$
\begin{aligned}
& \mathcal{D}_{0^{+}}^{\gamma} \phi_{p}\left(\mathcal{D}_{0^{+}}^{\epsilon} u(t)\right)=\psi_{1}(t, u(t)), \quad t \in \mathcal{J}^{\prime}, \\
& \Delta u\left(t_{k}\right)=\mathcal{I}_{k}\left(u\left(t_{k}\right)\right), \quad \Delta \phi_{p}\left(\mathcal{D}_{0^{+}}^{\epsilon} u\left(t_{k}\right)\right)=c_{k}, \quad k=1,2, \ldots, m, \\
& u(0)=u_{0},\left.\quad \mathcal{D}_{0^{+}}^{\epsilon} u(t)\right|_{t=0}=u_{1},
\end{aligned}
$$

where $\psi_{1}(t, u(t)), \mathcal{I}_{k}(u(t))$ are continuous real valued functions. $\mathcal{D}_{0^{+}}^{\gamma}, \mathcal{D}_{0^{+}}^{\epsilon}$, are Caputo's fractional derivatives of order $\gamma$ and $\epsilon$ where $0<\epsilon, \gamma \leq 1, \epsilon+\gamma \leq 2 . u_{0}, u_{1} \in \mathbb{R}, \mathcal{J}=[0,1]$, $t_{i} \leq t_{i+1}$ for $i=0, \ldots, m$, with $t_{m+1}=1, \mathcal{J}^{\prime}=\mathcal{J} \backslash\left\{t_{1}, \ldots, t_{m}\right\} . \Delta u\left(t_{k}\right)=u\left(t_{k}^{+}\right)-u\left(t_{k}^{-}\right)$, where $u\left(t_{k}^{+}\right), u\left(t_{k}^{-}\right)$are the right and left limits of $u(t)$ at $t=t_{k}(k=1,2, \ldots, m)$, respectively.

Feckan et al. [21] studied an impulsive FDE for the existence of solution of the kind:

$$
\begin{aligned}
& \mathcal{D}_{0, t}^{\epsilon} u(t)=\psi_{1}(t, u(t)), \quad t \in \mathcal{J}^{\prime}, \\
& u(0)=u_{0}, \quad \Delta u\left(t_{k}\right)=\mathcal{I}_{k}\left(u\left(t_{k}^{-}\right)\right), \quad k=1,2, \ldots, m,
\end{aligned}
$$

where $\psi_{1}(t, u(t))$, are continuous real valued functions, $\mathcal{I}_{k}: \mathbb{R} \rightarrow \mathbb{R}$. $\mathcal{D}_{0^{+}}^{\epsilon}$ is Caputo's fractional derivative of order $0<\epsilon<1 . u_{0} \in \mathbb{R}, \mathcal{J}=[0,1], t_{i} \leq t_{i+1}$ for $i=0, \ldots, m$, with $t_{m+1}=1$, $\mathcal{J}^{\prime}=\mathcal{J} \backslash\left\{t_{1}, \ldots, t_{m}\right\} . \Delta u\left(t_{k}\right)=u\left(t_{k}^{+}\right)-u\left(t_{k}^{-}\right)$, where $u\left(t_{k}^{+}\right), u\left(t_{k}^{-}\right)$are the right and left limits of $u(t)$ at $t=t_{k}(k=1,2, \ldots, m)$, respectively.

Inspired from the above research problems and the references given [22-26]. We apply a fixed point approach to examine EUS and Hyers-Ulam stability for the nonlinear impulsive fractional order DE:

$$
\left\{\begin{array}{l}
\mathcal{D}^{\gamma}\left[\phi_{p}\left[\mathcal{D}^{\epsilon} u(t)\right]\right]-\psi_{1}(t, u(t))=0, \\
\Delta\left[\phi_{p}\left[\mathcal{D}^{\epsilon} u\left(t_{k}\right)\right]\right]=b_{k}^{*}, \quad \Delta\left[\phi_{p}\left[\mathcal{D}^{\epsilon} u\left(t_{k}\right)\right]\right]^{\prime}=c_{k}^{*}, \quad \Delta u\left(t_{k}\right)=I_{k}\left(u\left(t_{k}\right)\right), \\
\phi_{p}\left(\mathcal{D}^{\epsilon} u(0)\right)=0, \quad \phi_{p}\left(\mathcal{D}^{\epsilon} u(1)\right)=\lambda^{*} \phi_{p}^{\prime}\left(\mathcal{D}^{\epsilon} u(1)\right), \quad u(0)=0
\end{array}\right.
$$


where $\psi_{1}$ is a continuous function. The orders are $\epsilon \in(0,1], \gamma \in(1,2] . \psi_{1} \in \mathcal{L}[0,1]$ and $\mathcal{D}^{\epsilon}$, $\mathcal{D}^{\gamma}$ are Caputo's fractional derivatives. The $p$-Laplacian operator $\phi_{p}$ satisfies $\phi_{p}(r)=|r|^{p-2} r$ where $1 / p+1 / q=1, \phi_{q}$ is the inverse of $\phi_{p}$. The problem (1.3) is more complicated and general than those considered earlier, given above (1.2).

Definition 1.1 The fractional integral for $\epsilon>0$ of $\psi:(0,+\infty) \rightarrow \mathbb{R}$ is given by

$$
\mathcal{I}^{\epsilon} \psi(t)=\frac{1}{\Gamma(\epsilon)} \int_{0}^{t}(t-s)^{\epsilon-1} \psi(s) d s
$$

provided that the integral on the right side is point-wise defined on the interval $(0,+\infty)$, where

$$
\Gamma(\epsilon)=\int_{0}^{+\infty} e^{-s} s^{\epsilon-1} d s
$$

Definition 1.2 The fractional derivative in Caputo's sense of order $\epsilon>0$, for continuous function $\psi(t):(0,+\infty) \rightarrow \mathbb{R}$ is given by

$$
\mathcal{D}^{\epsilon} \psi(t)=\frac{1}{\Gamma(k-\theta)} \int_{0}^{t}(t-s)^{k-\theta-1} \psi^{(k)}(s) d s,
$$

for $n=[\theta]+1$, where $[\theta]$ is used for the integer part of $\theta$, defined on $(0,+\infty)$.

Lemma 1.1 Let $\theta \in(n-1, n], \psi \in C^{n-1}$, then

$$
\mathcal{I}^{\theta} \mathcal{D}^{\theta} \psi(t)=\psi(t)+k_{0}+k_{1} t+k_{2} t^{2}+\cdots+k_{n-1} t^{n-1},
$$

for the $k_{i} \in \mathbb{R}$ for $i=0,1,2, \ldots, n-1$.

Lemma $1.2([13,15,27])$ For the $\phi_{p}$-operator, the following hold true:

(1) If $1<p \leq 2, \ell_{1}^{*} \ell_{2}^{*}>0$ and $\left|\ell_{1}^{*}\right|,\left|\ell_{2}^{*}\right| \geq \rho>0$, then

$$
\left|\phi_{p}\left(\ell_{1}^{*}\right)-\phi_{p}\left(\ell_{2}^{*}\right)\right| \leq(p-1) \rho^{p-2}\left|\ell_{1}^{*}-\ell_{2}^{*}\right|
$$

(2) If $p>2$, and $\left|\ell_{1}^{*}\right|,\left|\ell_{2}^{*}\right| \leq \rho^{*}$, then

$$
\left|\phi_{p}\left(\ell_{1}^{*}\right)-\phi_{p}\left(\ell_{2}^{*}\right)\right| \leq(p-1) \rho^{* p-2}\left|\ell_{1}^{*}-\ell_{2}^{*}\right| .
$$

Paper organization: In this paper, we aim to study EUS and the Hyers-Ulam stability for BVPs of impulsive fractional order DEs with $\phi_{p}$-operator (1.3) and to illustrate the results via an application. The paper is divided in five sections. The introduction part includes the basic and related literature in first section. The next section is reserved for the EUS for the impulsive fractional order DE with $p$-Laplacian operator (1.3). Hyers-Ulam stability is defined in the third section and it is verified that the impulsive fractional order DE (1.3) is Hyers-Ulam stable. The application of the results is given in Sect. 4. Finally, Sect. 5 is for the conclusion of the paper. The fractional order impulsive DE with $p$-Laplacian operator (1.3) is transferred into an integral equation with the help of a fractional integral operator 
and the properties of the $p$-Laplacian operator. With certain assumptions, the EUS for the (1.3) are proved and then the Hyers-Ulam stability is studied. An important example is also provided for application.

\section{Results for EUS}

Theorem 2.1 Assume that $\psi_{1} \in \mathcal{C}[0,1]$ be an integrable function satisfying (1.3). Then, for $\alpha \in(1,2], \gamma \in(0,1]$, the solution of the following impulsive FDE with a $p$-Laplacian operator:

$$
\left\{\begin{array}{l}
\mathcal{D}^{\gamma}\left[\phi_{p}\left[\mathcal{D}^{\epsilon} u(t)\right]\right]-\psi_{1}(t, u(t))=0, \\
\Delta\left[\phi_{p}\left[\mathcal{D}^{\epsilon} u\left(t_{k}\right)\right]\right]=b_{k}^{*}, \quad \Delta\left[\phi_{p}\left[\mathcal{D}^{\epsilon} u\left(t_{k}\right)\right]\right]^{\prime}=c_{k}^{*}, \quad \Delta u\left(t_{k}\right)=I_{k}\left(u\left(t_{k}\right)\right), \\
\phi_{p}\left(\mathcal{D}^{\epsilon} u(0)\right)=0, \quad \phi_{p}\left(\mathcal{D}^{\epsilon} u(1)\right)=\lambda^{*} \phi_{p}^{\prime}\left(\mathcal{D}^{\epsilon} u(1)\right), \quad u(0)=0
\end{array}\right.
$$

is

$$
\begin{aligned}
u(t)= & \frac{1}{\Gamma(\epsilon)} \int_{t_{k-1}}^{t}(t-\tau)^{\epsilon-1} \phi_{q}(\mathcal{G}(u(\tau))) d \tau+\frac{\sum_{k=1}^{m}}{\Gamma(\epsilon)} \int_{t_{k-1}}^{t_{k}}\left(t_{k}-\tau\right)^{\epsilon-1} \phi_{q}(\mathcal{G}(u(\tau))) d \tau \\
& +\sum_{k=1}^{m} I_{k}\left(u\left(t_{k}\right)\right),
\end{aligned}
$$

where

$$
\begin{aligned}
\mathcal{G}(u(t))= & \frac{1}{\Gamma(\gamma)} \int_{t_{1}}^{t}(t-s)^{\gamma-1} \psi_{1}(s, u(s)) d s+\frac{\sum_{k=1}^{m}}{\Gamma(\gamma)} \int_{t_{k-1}}^{t_{k}}\left(t_{k}-s\right)^{\gamma-1} \psi_{1}(s, u(s)) d s \\
& +\sum_{k=1}^{m} \frac{\left(t-t_{k}\right)}{\Gamma(\gamma-1)} \int_{t_{k-1}}^{t_{k}}\left(t_{1}-s\right)^{\gamma-2} \psi_{1}(s, u(s)) d s \\
& +\frac{t}{1-\lambda^{*}}\left[\frac{\lambda^{*}}{\Gamma(\gamma-1)} \int_{0}^{1}(1-s)^{\gamma-2} \psi_{1}(s, u(s)) d s\right. \\
& +\frac{\sum_{k=1}^{m}\left(\lambda^{*}-1+t_{k}\right)}{\Gamma(\gamma-1)} \int_{t_{k-1}}^{t_{k}}\left(t_{k}-s\right)^{\gamma-2} \psi_{1}(s, u(s)) d s \\
& -\frac{1}{\Gamma(\gamma)} \int_{0}^{1}(1-s)^{\gamma-1} \psi_{1}(s, u(s)) d s \\
& \left.-\frac{\sum_{k=}^{m}}{\Gamma(\gamma)} \int_{t_{k-1}}^{t_{k}}\left(t_{k}-s\right)^{\gamma-1} \psi_{1}(s, u(s)) d s+\sum_{k=1}^{m}\left(\lambda^{*}-1+t_{k}\right) c_{k}^{*}\right] \\
& +\sum_{k=1}^{m} b_{k}^{*}+\sum_{k=1}^{m}\left(t-t_{k}\right) c_{k}^{*} .
\end{aligned}
$$

Proof Let $u(t)=\phi_{p}\left[\mathcal{D}^{\epsilon} u(t)\right]$, then the problem (2.1) gets the form

$$
\left\{\begin{array}{l}
\mathcal{D}^{\gamma}[u(t)]-\psi_{1}(t, u(t))=0, \\
\Delta\left[u\left(t_{k}\right)\right]=b_{k}^{*}, \quad \Delta\left[u\left(t_{k}\right)\right]^{\prime}=c_{k}^{*} \\
u(0)=0, \quad u(1)=\lambda^{*} u^{\prime}(1) .
\end{array}\right.
$$


We apply the $\mathcal{I}^{\gamma}$-operator on (2.4) and with the help of Lemma 1.1, we have

$$
\begin{aligned}
u(t) & =\mathcal{I}^{\gamma}\left[\psi_{1}(t, u(t))\right]+c_{1}+c_{2} t \\
& =\frac{1}{\Gamma(\gamma)} \int_{t_{0}}^{t}(t-s)^{\gamma-1} \psi_{1}(s, u(s)) d s+c_{1}+c_{2} t .
\end{aligned}
$$

For $t \in\left[t_{1}, t_{2}\right]$, we have

$$
u(t)=\frac{1}{\Gamma(\gamma)} \int_{t_{1}}^{t}(t-s)^{\gamma-1} \psi_{1}(s, u(s)) d s+c_{3}+c_{4}\left(t-t_{1}\right) .
$$

From (2.5) and (2.6), we have

$$
u\left(t_{1}^{-}\right)=\frac{1}{\Gamma(\gamma)} \int_{t_{0}}^{t_{1}}\left(t_{1}-s\right)^{\gamma-1} \psi_{1}(s, u(s)) d s+c_{1}+c_{2} t_{1}, \quad u\left(t_{1}^{+}\right)=c_{3} .
$$

With the help of (2.4), we have

$$
\begin{aligned}
\Delta u\left(t_{1}\right) & =u\left(t_{1}^{+}\right)-u\left(t_{1}^{-}\right) \\
& =c_{3}-\frac{1}{\Gamma(\gamma)} \int_{t_{0}}^{t_{1}}\left(t_{1}-s\right)^{\gamma-1} \psi_{1}(s, u(s)) d s-c_{1}-c_{2} t_{1}=b_{1}^{*} .
\end{aligned}
$$

This implies

$$
c_{3}=\frac{1}{\Gamma(\gamma)} \int_{t_{0}}^{t_{1}}\left(t_{1}-s\right)^{\gamma-1} \psi_{1}(s, u(s)) d s+c_{1}+c_{2} t_{1}+b_{1}^{*} .
$$

For $t \in\left[t_{0}, t_{1}\right],(2.5)$ implies

$$
u^{\prime}(t)=\frac{1}{\Gamma(\gamma-1)} \int_{t_{0}}^{t}(t-s)^{\gamma-2} \psi_{1}(s, u(s)) d s+c_{2} .
$$

Therefore

$$
u^{\prime}\left(t_{1}^{-}\right)=\frac{1}{\Gamma(\gamma-1)} \int_{t_{0}}^{t_{1}}\left(t_{1}-s\right)^{\gamma-2} \psi_{1}(s, u(s)) d s+c_{2} .
$$

Similarly, we have $u^{\prime}\left(t_{1}^{+}\right)=c_{4}$. Using the condition $\Delta\left[x\left(t_{1}\right)\right]^{\prime}=c_{1}^{*}$, we have

$$
c_{4}=\frac{1}{\Gamma(\gamma-1)} \int_{t_{0}}^{t_{1}}\left(t_{1}-s\right)^{\gamma-2} \psi_{1}(s, u(s)) d s+c_{2}+c_{1}^{*} .
$$

With the help of (2.6), (2.9) and (2.12), we have

$$
\begin{aligned}
u(t)= & \frac{1}{\Gamma(\gamma)} \int_{t_{1}}^{t}(t-s)^{\gamma-1} \psi_{1}(s, u(s)) d s \\
& +\frac{1}{\Gamma(\gamma)} \int_{t_{0}}^{t_{1}}\left(t_{1}-s\right)^{\gamma-1} \psi_{1}(s, u(s)) d s+c_{1}+c_{2} t_{1}+I_{1}\left(u\left(t_{1}\right)\right) \\
& +\left(t-t_{1}\right)\left[\frac{1}{\Gamma(\gamma-1)} \int_{t_{0}}^{t_{1}}\left(t_{1}-s\right)^{\gamma-2} \psi_{1}(s, u(s)) d s+c_{2}+c_{1}^{*}\right]
\end{aligned}
$$




$$
\begin{aligned}
= & \frac{1}{\Gamma(\gamma)} \int_{t_{1}}^{t}(t-s)^{\gamma-1} \psi_{1}(s, u(s)) d s+\frac{1}{\Gamma(\gamma)} \int_{t_{0}}^{t_{1}}\left(t_{1}-s\right)^{\gamma-1} \psi_{1}(s, u(s)) d s \\
& +\frac{\left(t-t_{1}\right)}{\Gamma(\gamma-1)} \int_{t_{0}}^{t_{1}}\left(t_{1}-s\right)^{\gamma-2} \psi_{1}(s, u(s)) d s+c_{1}+c_{2} t+b_{1}^{*}+\left(t-t_{1}\right) c_{1}^{*} .
\end{aligned}
$$

For $t \in\left[t_{k-1}, t_{k}\right]$, we have

$$
\begin{aligned}
u(t)= & \frac{1}{\Gamma(\gamma)} \int_{t_{1}}^{t}(t-s)^{\gamma-1} \psi_{1}(s, u(s)) d s+\frac{\sum_{k=1}^{m}}{\Gamma(\gamma)} \int_{t_{k-1}}^{t_{k}}\left(t_{k}-s\right)^{\gamma-1} \psi_{1}(s, u(s)) d s \\
& +\sum_{k=1}^{m} \frac{\left(t-t_{k}\right)}{\Gamma(\gamma-1)} \int_{t_{k-1}}^{t_{k}}\left(t_{1}-s\right)^{\gamma-2} \psi_{1}(s, u(s)) d s+c_{1}+c_{2} t \\
& +\sum_{k=1}^{m} b_{k}^{*}+\sum_{k=1}^{m}\left(t-t_{k}\right) c_{k}^{*} .
\end{aligned}
$$

With the help of (2.5), (2.13) and the initial condition in (2.4) we get $c_{1}=0$ and

$$
\begin{aligned}
c_{2}= & \frac{1}{1-\lambda^{*}}\left[\frac{\lambda^{*}}{\Gamma(\gamma-1)} \int_{0}^{1}(1-s)^{\gamma-2} \psi_{1}(s, u(s)) d s\right. \\
& +\frac{\sum_{k=1}^{m}\left(\lambda^{*}-1+t_{k}\right)}{\Gamma(\gamma-1)} \int_{t_{k-1}}^{t_{k}}\left(t_{k}-s\right)^{\gamma-2} \psi_{1}(s, u(s)) d s \\
& -\frac{1}{\Gamma(\gamma)} \int_{0}^{1}(1-s)^{\gamma-1} \psi_{1}(s, u(s)) d s-\frac{\sum_{k=}^{m}}{\Gamma(\gamma)} \int_{t_{k-1}}^{t_{k}}\left(t_{k}-s\right)^{\gamma-1} \psi_{1}(s, u(s)) d s \\
& \left.+\sum_{k=1}^{m}\left(\lambda^{*}-1+t_{k}\right) c_{k}^{*}\right] .
\end{aligned}
$$

Ultimately, with the help of (2.14) and (2.15), we have

$$
\begin{aligned}
u(t)= & \frac{1}{\Gamma(\gamma)} \int_{t_{1}}^{t}(t-s)^{\gamma-1} \psi_{1}(s, u(s)) d s+\frac{\sum_{k=1}^{m}}{\Gamma(\gamma)} \int_{t_{k-1}}^{t_{k}}\left(t_{k}-s\right)^{\gamma-1} \psi_{1}(s, u(s)) d s \\
& +\sum_{k=1}^{m} \frac{\left(t-t_{k}\right)}{\Gamma(\gamma-1)} \int_{t_{k-1}}^{t_{k}}\left(t_{1}-s\right)^{\gamma-2} \psi_{1}(s, u(s)) d s \\
& +\frac{t}{1-\lambda^{*}}\left[\frac{\lambda^{*}}{\Gamma(\gamma-1)} \int_{0}^{1}(1-s)^{\gamma-2} \psi_{1}(s, u(s)) d s\right. \\
& +\frac{\sum_{k=1}^{m}\left(\lambda^{*}-1+t_{k}\right)}{\Gamma(\gamma-1)} \int_{t_{k-1}}^{t_{k}}\left(t_{k}-s\right)^{\gamma-2} \psi_{1}(s, u(s)) d s \\
& -\frac{1}{\Gamma(\gamma)} \int_{0}^{1}(1-s)^{\gamma-1} \psi_{1}(s, u(s)) d s \\
& \left.-\frac{\sum_{k=}^{m}}{\Gamma(\gamma)} \int_{t_{k-1}}^{t_{k}}\left(t_{k}-s\right)^{\gamma-1} \psi_{1}(s, u(s)) d s+\sum_{k=1}^{m}\left(\lambda^{*}-1+t_{k}\right) c_{k}^{*}\right] \\
& +\sum_{k=1}^{m} b_{k}^{*}+\sum_{k=1}^{m}\left(t-t_{k}\right) c_{k}^{*} .
\end{aligned}
$$


With the help of (2.1) and (2.3), we have

$$
D^{\epsilon} u(t)=\phi_{q}(\mathcal{G}(u(t))) .
$$

Applying the integral operator $I^{\epsilon}$ and using Lemma 1.1 on (2.17), where $\epsilon \in(0,1]$ and $t \in\left[t_{0}, t_{1}\right]$, we have

$$
u(t)=I^{\epsilon} \phi_{q}(\mathcal{G}(u(t)))+d_{1}=\frac{1}{\Gamma(\epsilon)} \int_{t_{0}}^{t}(t-\tau)^{\epsilon-1} \phi_{q}(\mathcal{G}(u(\tau))) d \tau+d_{1} .
$$

For $t \in\left[t_{1}, t_{2}\right],(2.18)$ implies

$$
u(t)=\frac{1}{\Gamma(\epsilon)} \int_{t_{1}}^{t}(t-\tau)^{\epsilon-1} \phi_{q}\left(\mathcal{G}(u(\tau)) d \tau+d_{2} .\right.
$$

Thus

$$
u\left(t_{1}^{-}\right)=\frac{1}{\Gamma(\epsilon)} \int_{t_{0}}^{t_{1}}\left(t_{1}-\tau\right)^{\epsilon-1} \phi_{q}\left(\mathcal{G}(u(\tau)) d \tau+d_{1}, \quad u\left(t_{1}^{+}\right)=d_{2} .\right.
$$

With the help of the condition $\Delta u\left(t_{1}\right)=I_{1}\left(u\left(t_{1}\right)\right)$ and (2.20), we get

$$
d_{2}=\frac{1}{\Gamma(\epsilon)} \int_{t_{0}}^{t_{1}}\left(t_{1}-\tau\right)^{\epsilon-1} \phi_{q}\left(\mathcal{G}(u(\tau)) d \tau+d_{1}+I_{1}\left(u\left(t_{1}\right)\right) .\right.
$$

From (2.19) and (2.21), we have

$$
\begin{aligned}
u(t)= & \frac{1}{\Gamma(\epsilon)} \int_{t_{0}}^{t}(t-\tau)^{\epsilon-1} \phi_{q}(\mathcal{G}(u(\tau))) d \tau+\frac{1}{\Gamma(\epsilon)} \int_{t_{0}}^{t_{1}}\left(t_{1}-\tau\right)^{\epsilon-1} \phi_{q}(\mathcal{G}(u(\tau))) d \tau \\
& +d_{1}+I\left(u\left(t_{1}\right)\right) .
\end{aligned}
$$

For $t \in\left[t_{k-1}, t_{k}\right]$, we have

$$
\begin{aligned}
u(t)= & \int_{t_{k-1}}^{t} \frac{(t-\tau)^{\epsilon-1}}{\Gamma(\epsilon)} \phi_{q}(\mathcal{G}(u(\tau))) d \tau+\frac{\sum_{k=1}^{m}}{\Gamma(\epsilon)} \int_{t_{k-1}}^{t_{k}}\left(t_{k}-\tau\right)^{\epsilon-1} \phi_{q}(\mathcal{G}(u(\tau))) d \tau \\
& +d_{1}+\sum_{k=1}^{m} I_{k}\left(u\left(t_{k}\right)\right) .
\end{aligned}
$$

With the initial condition $u(0)=0$ and (2.18), we have $d_{1}=0$. Consequently, we have

$$
\begin{aligned}
u(t)= & \frac{1}{\Gamma(\epsilon)} \int_{t_{k-1}}^{t}(t-\tau)^{\epsilon-1} \phi_{q}(\mathcal{G}(u(\tau))) d \tau \\
& +\frac{\sum_{k=1}^{m}}{\Gamma(\epsilon)} \int_{t_{k-1}}^{t_{k}}\left(t_{k}-\tau\right)^{\epsilon-1} \phi_{q}(\mathcal{G}(u(\tau))) d \tau+\sum_{k=1}^{m} I_{k}\left(u\left(t_{k}\right)\right) .
\end{aligned}
$$


We define an operator $\mathcal{F}^{*}: \mathcal{B} \rightarrow \mathcal{B}$ by

$$
\begin{aligned}
\mathcal{F}^{*}(u((t)))= & \frac{1}{\Gamma(\epsilon)} \int_{t_{k-1}}^{t}(t-\tau)^{\epsilon-1} \phi_{q}(\mathcal{G}(u(\tau))) d \tau \\
& +\frac{\sum_{k=1}^{m}}{\Gamma(\epsilon)} \int_{t_{k-1}}^{t_{k}}\left(t_{k}-\tau\right)^{\epsilon-1} \phi_{q}(\mathcal{G}(u(\tau))) d \tau+\sum_{k=1}^{m} I_{k}\left(u\left(t_{k}\right)\right),
\end{aligned}
$$

where $\mathcal{G}$ is defined in (2.3).

Theorem 2.2 Assume that conditions $\left(\mathcal{R}_{1}\right)-\left(\mathcal{R}_{3}\right)$ hold true. Then the operator $\mathcal{F}^{*}$ is continuous and satisfies the inequality

$$
\begin{aligned}
\left|\mathcal{F}^{*} u(t)\right| \leq & \frac{m+1}{\Gamma(\gamma+1)} \phi_{q}\left(\left(\frac{1}{\Gamma(\gamma+1)}+\frac{m}{\Gamma(\gamma)}+\frac{1}{1-\lambda^{*}}\left[\frac{\lambda^{*}(1+m)}{\Gamma(\gamma)}+\frac{1+m}{\Gamma(1+\gamma)}\right]\right) \mathcal{M}_{0}\right. \\
& \left.+\frac{m \lambda^{*} C^{*}}{1-\lambda^{*}}+B^{*}+C^{*}\right)+m \mathcal{M}_{1} .
\end{aligned}
$$

Proof Consider a convergent sequence $\left\{u_{n}\right\} \rightarrow u$ in a bounded set $\rho_{r}=\{u \in \mathcal{B}:\|u\| \leq r\}$. We show that $\left\|\mathcal{F}^{*}\left(u_{n}\right)-\mathcal{F}^{*}(u)\right\|$ goes to 0 as $n$ approaches $+\infty$. For this purpose, with the help of Lemma 1.2, we have

$$
\begin{aligned}
\left|\mathcal{F}^{*}\left(u_{n}\right)-\mathcal{F}^{*}(u)\right| & \\
\leq & (q-1) m^{q-2} \int_{t_{k-1}}^{t} \frac{(t-\tau)^{\epsilon-1}}{\Gamma(\epsilon)}\left[\frac{1}{\Gamma(\gamma)} \int_{\tau_{1}}^{\tau}(\tau-s)^{\gamma-1}\left|\psi_{1}\left(s, u_{n}(s)\right)-\psi_{1}(s, u(s))\right| d s\right. \\
& +\frac{\sum_{k=1}^{m}}{\Gamma(\gamma)} \int_{\tau_{k-1}}^{\tau_{k}}\left(\tau_{k}-s\right)^{\gamma-1}\left|\psi_{1}\left(s, u_{n}(s)\right)-\psi_{1}(s, u(s))\right| d s \\
& +\sum_{k=1}^{m} \frac{\left(\tau-\tau_{k}\right)}{\Gamma(\gamma-1)} \int_{\tau_{k-1}}^{\tau_{k}}\left(\tau_{1}-s\right)^{\gamma-2}\left|\psi_{1}\left(s, u_{n}(s)\right)-\psi_{1}(s, u(s))\right| d s \\
& +\frac{\tau}{1-\lambda^{*}}\left[\frac{\lambda^{*}}{\Gamma(\gamma-1)} \int_{0}^{1}(1-s)^{\gamma-2}\left|\psi_{1}\left(s, u_{n}(s)\right)-\psi_{1}(s, u(s))\right| d s\right. \\
& +\frac{\sum_{k=1}^{m}\left(\lambda^{*}-1+\tau_{k}\right)}{\Gamma(\gamma-1)} \int_{\tau_{k-1}}^{\tau_{k}}\left(\tau_{k}-s\right)^{\gamma-2}\left|\psi_{1}\left(s, u_{n}(s)\right)-\psi_{1}(s, u(s))\right| d s \\
& +\frac{1}{\Gamma(\gamma)} \int_{0}^{1}(1-s)^{\gamma-1}\left|\psi_{1}\left(s, u_{n}(s)\right)-\psi_{1}(s, u(s))\right| d s \\
& \left.\left.+\frac{\sum_{k=}^{m}}{\Gamma(\gamma)} \int_{\tau_{k-1}}^{\tau_{k}}\left(\tau_{k}-s\right)^{\gamma-1}\left|\psi_{1}\left(s, u_{n}(s)\right)-\psi_{1}(s, u(s))\right| d s\right]\right] d \tau \\
& +(q-1) m^{q-2} \frac{\sum_{k=1}^{m}}{\Gamma(\epsilon)} \int_{t_{k-1}}^{t_{k}}\left(t_{k}-\tau\right)^{\epsilon-1} \\
& \times\left[\frac{1}{\Gamma(\gamma)} \int_{\tau_{1}}^{\tau}(\tau-s)^{\gamma-1}\left|\psi_{1}\left(s, u_{n}(s)\right)-\psi_{1}(s, u(s))\right| d s\right. \\
+ & \frac{\sum_{k=1}^{m}}{\Gamma(\gamma)} \int_{\tau_{k-1}}^{\tau_{k}}\left(\tau_{k}-s\right)^{\gamma-1}\left|\psi_{1}\left(s, u_{n}(s)\right)-\psi_{1}(s, u(s))\right| d s
\end{aligned}
$$




$$
\begin{aligned}
& +\sum_{k=1}^{m} \frac{\left(\tau-\tau_{k}\right)}{\Gamma(\gamma-1)} \int_{\tau_{k-1}}^{\tau_{k}}\left(\tau_{1}-s\right)^{\gamma-2}\left|\psi_{1}\left(s, u_{n}(s)\right)-\psi_{1}(s, u(s))\right| d s \\
& +\frac{\tau}{1-\lambda^{*}}\left[\frac{\lambda^{*}}{\Gamma(\gamma-1)} \int_{0}^{1}(1-s)^{\gamma-2}\left|\psi_{1}\left(s, u_{n}(s)\right)-\psi_{1}(s, u(s))\right| d s\right. \\
& +\frac{\sum_{k=1}^{m}\left(\lambda^{*}-1+\tau_{k}\right)}{\Gamma(\gamma-1)} \int_{\tau_{k-1}}^{\tau_{k}}\left(\tau_{k}-s\right)^{\gamma-2}\left|\psi_{1}\left(s, u_{n}(s)\right)-\psi_{1}(s, u(s))\right| d s \\
& +\frac{1}{\Gamma(\gamma)} \int_{0}^{1}(1-s)^{\gamma-1}\left|\psi_{1}\left(s, u_{n}(s)\right)-\psi_{1}(s, u(s))\right| d s \\
& \left.\left.+\frac{\sum_{k=1}^{m}}{\Gamma(\gamma)} \int_{\tau_{k-1}}^{\tau_{k}}\left(\tau_{k}-s\right)^{\gamma-1}\left|\psi_{1}\left(s, u_{n}(s)\right)-\psi_{1}(s, u(s))\right| d s\right]\right] d \tau \\
& +\sum_{k=1}^{m} I_{k}\left(\left|u_{n}\left(t_{k}\right)-u\left(t_{k}\right)\right|\right) .
\end{aligned}
$$

By (2.26), and continuity of $\psi_{1}$, we have $\left|\mathcal{F}^{*} u_{n}-\mathcal{F}^{*} u\right|$ goes to 0 as $n$ approaches $+\infty$. This implies that $\mathcal{F}^{*}$ is continuous. Next, consider a bounded subset $\mathcal{B} \subset \mathcal{L}$. Also, with the help of continuity of the functions $f$ and $\mathcal{I}_{k}$, we have some constants, say, $\mathcal{M}_{0}, \mathcal{M}_{1}>0$, such that $\left|\psi_{1}(t, u(t))\right| \leq \mathcal{M}_{0},\left|\mathcal{I}_{k}\left(u\left(t_{k}\right)\right)\right| \leq \mathcal{M}_{1}$ for $k=1,2, \ldots, m$ for $t \in \mathcal{J}=[0,1]$ and $u \in \mathcal{B}$. Then, for the boundedness of $\mathcal{F}^{*}$, we have

$$
\begin{aligned}
& \left|\mathcal{F}^{*} u(t)\right| \\
& =\mid \frac{1}{\Gamma(\epsilon)} \int_{t_{k-1}}^{t}(t-\tau)^{\epsilon-1} \phi_{q}\left(\frac{1}{\Gamma(\gamma)} \int_{\tau_{1}}^{\tau}(\tau-s)^{\gamma-1} \psi_{1}(s, u(s)) d s\right. \\
& +\frac{\sum_{k=1}^{m}}{\Gamma(\gamma)} \int_{\tau_{k-1}}^{\tau_{k}}\left(\tau_{k}-s\right)^{\gamma-1} \psi_{1}(s, u(s)) d s+\sum_{k=1}^{m} \frac{\left(\tau-\tau_{k}\right)}{\Gamma(\gamma-1)} \int_{\tau_{k-1}}^{\tau_{k}}\left(\tau_{1}-s\right)^{\gamma-2} \psi_{1}(s, u(s)) d s \\
& +\frac{\tau}{1-\lambda^{*}}\left[\frac{\lambda^{*}}{\Gamma(\gamma-1)} \int_{0}^{1}(1-s)^{\gamma-2} \psi_{1}(s, u(s)) d s\right. \\
& +\frac{\sum_{k=1}^{m}\left(\lambda^{*}-1+\tau_{k}\right)}{\Gamma(\gamma-1)} \int_{\tau_{k-1}}^{\tau_{k}}\left(\tau_{k}-s\right)^{\gamma-2} \psi_{1}(s, u(s)) d s \\
& -\frac{1}{\Gamma(\gamma)} \int_{0}^{1}(1-s)^{\gamma-1} \psi_{1}(s, u(s)) d s \\
& \left.-\frac{\sum_{k=}^{m}}{\Gamma(\gamma)} \int_{\tau_{k-1}}^{\tau_{k}}\left(\tau_{k}-s\right)^{\gamma-1} \psi_{1}(s, u(s)) d s+\sum_{k=1}^{m}\left(\lambda^{*}-1+\tau_{k}\right) c_{k}^{*}\right] \\
& \left.+\sum_{k=1}^{m} b_{k}^{*}+\sum_{k=1}^{m}\left(\tau-\tau_{k}\right) c_{k}^{*}\right) d \tau \\
& +\frac{\sum_{k=1}^{m}}{\Gamma(\epsilon)} \int_{t_{k-1}}^{t_{k}}\left(t_{k}-\tau\right)^{\epsilon-1} \phi_{q}\left(\frac{1}{\Gamma(\gamma)} \int_{\tau_{1}}^{\tau}(\tau-s)^{\gamma-1} \psi_{1}(s, u(s)) d s\right. \\
& +\frac{\sum_{k=1}^{m}}{\Gamma(\gamma)} \int_{\tau_{k-1}}^{\tau_{k}}\left(\tau_{k}-s\right)^{\gamma-1} \psi_{1}(s, u(s)) d s \\
& +\sum_{k=1}^{m} \frac{\left(\tau-\tau_{k}\right)}{\Gamma(\gamma-1)} \int_{\tau_{k-1}}^{\tau_{k}}\left(\tau_{1}-s\right)^{\gamma-2} \psi_{1}(s, u(s)) d s
\end{aligned}
$$




$$
\begin{aligned}
& +\frac{t}{1-\lambda^{*}}\left[\frac{\lambda^{*}}{\Gamma(\gamma-1)} \int_{0}^{1}(1-s)^{\gamma-2} \psi_{1}(s, u(s)) d s\right. \\
& +\frac{\sum_{k=1}^{m}\left(\lambda^{*}-1+\tau_{k}\right)}{\Gamma(\gamma-1)} \int_{\tau_{k-1}}^{\tau_{k}}\left(\tau_{k}-s\right)^{\gamma-2} \psi_{1}(s, u(s)) d s \\
& -\frac{1}{\Gamma(\gamma)} \int_{0}^{1}(1-s)^{\gamma-1} \psi_{1}(s, u(s)) d s \\
& \left.-\frac{\sum_{k=}^{m}}{\Gamma(\gamma)} \int_{\tau_{k-1}}^{\tau_{k}}\left(\tau_{k}-s\right)^{\gamma-1} \psi_{1}(s, u(s)) d s+\sum_{k=1}^{m}\left(\lambda^{*}-1+\tau_{k}\right) c_{k}^{*}\right] \\
& \left.+\sum_{k=1}^{m} b_{k}^{*}+\sum_{k=1}^{m}\left(\tau-\tau_{k}\right) c_{k}^{*}\right) d \tau+\sum_{k=1}^{m} I_{k}\left(u\left(t_{k}\right)\right) \mid \\
\leq & \frac{1}{\Gamma(\epsilon+1)} \phi_{q}\left(\left(\frac{1}{\Gamma(\gamma+1)}+\frac{m}{\Gamma(\gamma+1)}+\frac{m}{\Gamma(\gamma)}+\frac{1}{1-\lambda^{*}}\left[\frac{\lambda^{*}}{\Gamma(\gamma)}+\frac{m \lambda^{*}}{\Gamma(\gamma)}+\frac{1}{\Gamma(\gamma+1)}\right.\right.\right. \\
& \left.\left.\left.+\frac{m}{\Gamma(\gamma+1)}\right]\right) \mathcal{M}_{0}+\frac{m \lambda^{*} C^{*}}{1-\lambda^{*}}+B^{*}+C^{*}\right)+\frac{m}{\Gamma(\epsilon+1)} \phi_{q}\left(\left(\frac{1}{\Gamma(\gamma+1)}+\frac{m}{\Gamma(\gamma+1)}\right.\right. \\
& \left.+\frac{m}{\Gamma(\gamma)}+\frac{1}{1-\lambda^{*}}\left[\frac{\lambda^{*}}{\Gamma(\gamma)}+\frac{m \lambda^{*}}{\Gamma(\gamma)}+\frac{1}{\Gamma(\gamma+1)}+\frac{m}{\Gamma(\gamma+1)}\right]\right) \mathcal{M}_{0} \\
& \left.+\frac{m \lambda^{*} C^{*}}{1-\lambda^{*}}+B^{*}+C^{*}\right)+m \mathcal{M}_{1} \\
= & \frac{m+1}{\Gamma(\gamma+1)} \phi_{q}\left(\left(\frac{1}{\Gamma(\gamma+1)}+\frac{m}{\Gamma(\gamma)}+\frac{1}{1-\lambda^{*}}\left[\frac{\lambda^{*}(1+m)}{\Gamma(\gamma)}+\frac{1+m}{\Gamma(1+\gamma)}\right]\right) \mathcal{M}_{0}\right. \\
& \left.+\frac{m \lambda^{*} C^{*}}{1-\lambda^{*}}+B^{*}+C^{*}\right)+m \mathcal{M} 1 .
\end{aligned}
$$

For the EUS theorems, we consider the assumptions:

- $\left(\mathcal{R}_{1}\right) \psi_{1}(t, u(t)): \mathcal{J} \times \mathbb{R} \rightarrow \mathbb{R}$ is a bounded continuous, that is, there exists some constant $\mathcal{M}_{0}>0$, such that $\left|\psi_{1}(t, u(t))\right| \leq \mathcal{M}_{0}$.

- $\left(\mathcal{R}_{2}\right)$ For some constants $\mathcal{L}_{\psi_{1}}>0$, we have

$$
\left|\psi_{1}(t, u(t))-\psi_{1}(t, \bar{u}(t))\right| \leq \mathcal{L}_{\psi_{1}}|u(t)-\bar{u}(t)| .
$$

- $\left(\mathcal{R}_{3}\right)$ For some constant $\mathcal{L}>0$, we have

$$
\left\|\mathcal{I}_{k} u\left(t_{k}\right)-\overline{\mathcal{I}}_{k} u\left(t_{k}\right)\right\| \leq \mathcal{J}\|u-\bar{u}\|
$$

Theorem 2.3 With assumption $\left(\mathcal{R}_{1}\right), \mathcal{F}^{*}: \mathcal{L} \rightarrow \mathcal{L}$ is compact and $\xi$-Lipschitz with constant zero.

Proof With the help of Theorem 2.2, the operator $\mathcal{F}^{*}$ is bounded. Next, with the help of (2.3), (2.25), (2.28) and Lemma 1.2 for any $t_{1}, t_{2} \in[0,1]$, and we have

$$
\begin{aligned}
& \left|\mathcal{F}^{*} u\left(t_{2}\right)-\mathcal{F}^{*} u\left(t_{1}\right)\right| \\
& \quad \leq\left|\frac{1}{\Gamma(\epsilon)} \int_{t_{k-1}}^{t_{2}}\left(t_{2}-\tau\right)^{\epsilon-1} \phi_{q}(\mathcal{G}(u(\tau))) d \tau-\frac{1}{\Gamma(\epsilon)} \int_{t_{k-1}}^{t_{1}}\left(t_{1}-\tau\right)^{\epsilon-1} \phi_{q}(\mathcal{G}(u(\tau))) d \tau\right|
\end{aligned}
$$




$$
\begin{aligned}
\leq & \frac{1}{\Gamma(\epsilon)}\left|\int_{t_{k-1}}^{t_{2}}\left(t_{2}-\tau\right)^{\epsilon-1}-\int_{t_{k-1}}^{t_{1}}\left(t_{1}-\tau\right)^{\epsilon-1}\right| \phi_{q}|\mathcal{G}(u(\tau))| d \tau \\
\leq & \frac{1}{\Gamma(\epsilon)}\left|\int_{t_{k-1}}^{t_{2}}(t-\tau)^{\epsilon-1}-\int_{t_{k-1}}^{t_{1}}\left(t_{1}-\tau\right)^{\epsilon-1}\right| \phi_{q} \mid \frac{1}{\Gamma(\gamma)} \int_{\tau_{1}}^{\tau}(\tau-s)^{\gamma-1} \psi_{1}(s, u(s)) d s \\
& +\frac{\sum_{k=1}^{m}}{\Gamma(\gamma)} \int_{\tau_{k-1}}^{\tau_{k}}\left(\tau_{k}-s\right)^{\gamma-1} \psi_{1}(s, u(s)) d s+\sum_{k=1}^{m} \frac{\left(\tau-\tau_{k}\right)}{\Gamma(\gamma-1)} \int_{\tau_{k-1}}^{\tau_{k}}\left(\tau_{1}-s\right)^{\gamma-2} \psi_{1}(s, u(s)) d s \\
& +\frac{\tau}{1-\lambda^{*}}\left[\frac{\lambda^{*}}{\Gamma(\gamma-1)} \int_{0}^{1}(1-s)^{\gamma-2} \psi_{1}(s, u(s)) d s\right. \\
& +\frac{\sum_{k=1}^{m}\left(\lambda^{*}-1+\tau_{k}\right)}{\Gamma(\gamma-1)} \int_{\tau_{k-1}}^{\tau_{k}}\left(\tau_{k}-s\right)^{\gamma-2} \psi_{1}(s, u(s)) d s \\
& -\frac{1}{\Gamma(\gamma)} \int_{0}^{1}(1-s)^{\gamma-1} \psi_{1}(s, u(s)) d s-\frac{\sum_{k=1}^{m}}{\Gamma(\gamma)} \int_{\tau_{k-1}}^{\tau_{k}}\left(\tau_{k}-s\right)^{\gamma-1} \psi_{1}(s, u(s)) d s \\
& \left.+\sum_{k=1}^{m}\left(\lambda^{*}-1+\tau_{k}\right) c_{k}^{*}\right]+\sum_{k=1}^{m} b_{k}^{*}+\sum_{k=1}^{m}\left(t-t_{k}\right) c_{k}^{*} \mid d \tau \\
\leq & \frac{1}{\Gamma(\epsilon)}\left|\int_{0}^{t_{2}}(t-\tau)^{\epsilon-1}-\int_{0}^{t_{1}}\left(t_{1}-\tau\right)^{\epsilon-1}\right| \phi_{q}\left[\left(\frac{1+m}{\Gamma(\gamma+1)}+\frac{1}{1-\lambda^{*}}\left[\frac{\lambda^{*}(1+m)}{\Gamma(\gamma)}\right.\right.\right. \\
& \left.\left.\left.+\frac{1+m}{\Gamma(1+\gamma)}\right]\right)(k+\|u\|)+\frac{m \lambda^{*} C^{*}}{1-\lambda^{*}}+B^{*}+C^{*}\right] d \tau \\
\leq & \frac{t_{2}-t_{1}}{\Gamma(\epsilon+1)}\left[\left(\frac{1+m}{\Gamma(\gamma+1)}+\frac{1}{1-\lambda^{*}}\left[\frac{\lambda^{*}(1+m)}{\Gamma(\gamma)}+\frac{1+m}{\Gamma(1+\gamma)}\right]\right)(k+\|u\|)\right. \\
& \left.+\frac{m \lambda^{*} C^{*}}{1-\lambda^{*}}+B^{*}+C^{*}\right]^{q-1} \cdot
\end{aligned}
$$

As $t_{1} \rightarrow t_{2}$, (2.31) implies that $\left|\mathcal{F}^{*} u\left(t_{2}\right)-\mathcal{F}^{*} u\left(t_{1}\right)\right| \rightarrow 0$. Thus, $\mathcal{F}^{*}$ is equicontinuous and by Arzela-Ascoli's theorem, $\mathcal{F}^{*}$ is compact. Consequently, $\mathcal{F}^{*}$ is $\xi$-Lipschitz with constant 0 .

Theorem 2.4 Assume that the conditions $\left(\mathcal{R}_{1}\right),\left(\mathcal{R}_{2}\right)$ are true. Then the impulsive fractional order DE with $\phi_{p}$ (1.3) has a unique solution with assumption $\Delta^{*}<1$ for

$$
\begin{aligned}
\Delta^{*}= & {\left[(q-1) \mathcal{M}^{q-2} \frac{m+1}{\Gamma(\epsilon+1)}\left(\frac{1+m}{\Gamma(\gamma+1)}+\frac{1}{1-\lambda^{*}}\left[\frac{\lambda^{*}(1+m)}{\Gamma(\gamma)}+\frac{1+m}{\Gamma(1+\gamma)}\right]\right) \mathcal{L}_{\psi_{1}}\right.} \\
& +m \mathcal{J}] .
\end{aligned}
$$

Proof With the assumption of conditions $\left(\mathcal{R}_{1}\right),\left(\mathcal{R}_{2}\right)$, and Lemma 1.2, we have

$$
\begin{aligned}
& \left|\mathcal{F}^{*} u(t)-\mathcal{F}^{*} \bar{u}(t)\right| \\
& \leq \frac{1}{\Gamma(\epsilon)} \int_{t_{k-1}}^{t}(t-\tau)^{\epsilon-1}\left|\phi_{q}(\mathcal{G}(u(\tau)))-\phi_{q}(\mathcal{G}(\bar{u}(\tau)))\right| d \tau \\
& \quad+\frac{\sum_{k=1}^{m}}{\Gamma(\epsilon)} \int_{t_{k-1}}^{t_{k}}\left(t_{k}-\tau\right)^{\epsilon-1}\left|\phi_{q}(\mathcal{G}(u(\tau)))-\phi_{q}(\mathcal{G}(\bar{u}(\tau)))\right| d \tau
\end{aligned}
$$


Khan et al. Advances in Difference Equations

(2019) 2019:18

Page 12 of 16

$$
\begin{aligned}
& +\sum_{k=1}^{m}\left|I_{k}\left(u\left(t_{k}\right)\right)-I_{k}\left(\bar{u}\left(t_{k}\right)\right)\right| \\
& \leq(q-1) \mathcal{M}^{q-2} \frac{1}{\Gamma(\epsilon)} \int_{t_{k-1}}^{t}(t-\tau)^{\epsilon-1}\left(\frac{1}{\Gamma(\gamma)} \int_{\tau_{1}}^{\tau}(\tau-s)^{\gamma-1}\left|\psi_{1}(s, u(s))-\psi_{1}(s, \bar{u}(s))\right| d s\right. \\
& +\frac{\sum_{k=1}^{m}}{\Gamma(\gamma)} \int_{\tau_{k-1}}^{\tau_{k}}\left(\tau_{k}-s\right)^{\gamma-1}\left|\psi_{1}(s, u(s))-\psi_{1}(s, \bar{u}(s))\right| d s \\
& +\sum_{k=1}^{m} \frac{\left(t-\tau_{k}\right)}{\Gamma(\gamma-1)} \int_{\tau_{k-1}}^{\tau_{k}}\left(t_{1}-s\right)^{\gamma-2}\left|\psi_{1}(s, u(s))-\psi_{1}(s, \bar{u}(s))\right| d s \\
& +\frac{\tau}{1-\lambda^{*}}\left[\frac{\lambda^{*}}{\Gamma(\gamma-1)} \int_{0}^{1}(1-s)^{\gamma-2}\left|\psi_{1}(s, u(s))-\psi_{1}(s, \bar{u}(s))\right| d s\right. \\
& +\frac{\sum_{k=1}^{m}\left(\lambda^{*}-1+\tau_{k}\right)}{\Gamma(\gamma-1)} \int_{\tau_{k-1}}^{\tau_{k}}\left(\tau_{k}-s\right)^{\gamma-2}\left|\psi_{1}(s, u(s))-\psi_{1}(s, \bar{u}(s))\right| d s \\
& +\frac{1}{\Gamma(\gamma)} \int_{0}^{1}(1-s)^{\gamma-1}\left|\psi_{1}(s, u(s))-\psi_{1}(s, \bar{u}(s))\right| d s \\
& \left.\left.+\frac{\sum_{k=}^{m}}{\Gamma(\gamma)} \int_{\tau_{k-1}}^{\tau_{k}}\left(\tau_{k}-s\right)^{\gamma-1}\left|\psi_{1}(s, u(s))-\psi_{1}(s, \bar{u}(s))\right| d s\right]\right) d \tau \\
& +(q-1) \mathcal{M}^{q-2} \frac{\sum_{k=1}^{m}}{\Gamma(\epsilon)} \int_{t_{k-1}}^{t_{k}}\left(t_{k}-\tau\right)^{\epsilon-1} \\
& \times\left(\frac{1}{\Gamma(\gamma)} \int_{\tau_{1}}^{\tau}(\tau-s)^{\gamma-1}\left|\psi_{1}(s, u(s))-\psi_{1}(s, \bar{u}(s))\right| d s\right. \\
& +\frac{\sum_{k=1}^{m}}{\Gamma(\gamma)} \int_{\tau_{k-1}}^{\tau_{k}}\left(\tau_{k}-s\right)^{\gamma-1}\left|\psi_{1}(s, u(s))-\psi_{1}(s, \bar{u}(s))\right| d s \\
& +\sum_{k=1}^{m} \frac{\left(\tau-\tau_{k}\right)}{\Gamma(\gamma-1)} \int_{\tau_{k-1}}^{\tau_{k}}\left(\tau_{1}-s\right)^{\gamma-2}\left|\psi_{1}(s, u(s))-\psi_{1}(s, \bar{u}(s))\right| d s \\
& +\frac{\tau}{1-\lambda^{*}}\left[\frac{\lambda^{*}}{\Gamma(\gamma-1)} \int_{0}^{1}(1-s)^{\gamma-2}\left|\psi_{1}(s, u(s))-\psi_{1}(s, \bar{u}(s))\right| d s\right. \\
& +\frac{\sum_{k=1}^{m}\left(\lambda^{*}-1+\tau_{k}\right)}{\Gamma(\gamma-1)} \int_{\tau_{k-1}}^{\tau_{k}}\left(\tau_{k}-s\right)^{\gamma-2}\left|\psi_{1}(s, u(s))-\psi_{1}(s, \bar{u}(s))\right| d s \\
& +\frac{1}{\Gamma(\gamma)} \int_{0}^{1}(1-s)^{\gamma-1}\left|\psi_{1}(s, u(s))-\psi_{1}(s, \bar{u}(s))\right| d s \\
& \left.\left.+\frac{\sum_{k=}^{m}}{\Gamma(\gamma)} \int_{\tau_{k-1}}^{\tau_{k}}\left(\tau_{k}-s\right)^{\gamma-1}\left|\psi_{1}(s, u(s))-\psi_{1}(s, \bar{u}(s))\right| d s\right]\right) d \tau \\
& +\sum_{k=1}^{m}\left|I_{k}\left(u\left(t_{k}\right)\right)-I_{k}\left(\bar{u}\left(t_{k}\right)\right)\right| \\
& \leq \frac{(q-1) \mathcal{M}^{q-2}}{\Gamma(\epsilon+1)}\left[\left(\frac{1+m}{\Gamma(\gamma+1)}+\frac{1}{1-\lambda^{*}}\left[\frac{\lambda^{*}(1+m)}{\Gamma(\gamma)}+\frac{1+m}{\Gamma(1+\gamma)}\right]\right) \mathcal{L}_{\psi_{1}}|u-\bar{u}|\right] \\
& +\frac{m(q-1) \mathcal{M}^{q-2}}{\Gamma(\epsilon+1)}\left[\left(\frac{1+m}{\Gamma(\gamma+1)}+\frac{1}{1-\lambda^{*}}\left[\frac{\lambda^{*}(1+m)}{\Gamma(\gamma)}+\frac{1+m}{\Gamma(1+\gamma)}\right]\right) \mathcal{L}_{\psi_{1}}|u-\bar{u}|\right] \\
& +m \mathcal{J}|u-\bar{u}|
\end{aligned}
$$




$$
\begin{aligned}
\leq & {\left[(q-1) \mathcal{M}^{q-2} \frac{m+1}{\Gamma(\epsilon+1)}\left(\frac{1+m}{\Gamma(\gamma+1)}+\frac{1}{1-\lambda^{*}}\left[\frac{\lambda^{*}(1+m)}{\Gamma(\gamma)}+\frac{1+m}{\Gamma(1+\gamma)}\right]\right) \mathcal{L}_{\psi_{1}}\right.} \\
& +m \mathcal{J}]\|u-\bar{u}\| .
\end{aligned}
$$

With the assumption given in (2.32), and Banach's fixed point theorem, the operator $\mathcal{F}^{*}$ has a unique fixed point and is a unique solution of (1.3).

\section{Hyers-Ulam stability}

Here, we prove that (1.3) is Hyers-Ulam stable. We give the following definition for the stability.

Definition 3.1 The integral equation (2.24) is said to be Hyers-Ulam stable if for some fixed constant value $\lambda^{*}>0$,

$$
\begin{aligned}
\mid u(t) & -\frac{1}{\Gamma(\epsilon)} \int_{t_{k-1}}^{t}(t-\tau)^{\epsilon-1} \phi_{q}(\mathcal{G}(u(\tau))) d \tau \\
& -\frac{\sum_{k=1}^{m}}{\Gamma(\epsilon)} \int_{t_{k-1}}^{t_{k}}\left(t_{k}-\tau\right)^{\epsilon-1} \phi_{q}(\mathcal{G}(u(\tau))) d \tau-\sum_{k=1}^{m} I_{k}\left(u\left(t_{k}\right)\right) \mid \\
\leq & \lambda^{*}
\end{aligned}
$$

there exists a continuous function $u^{*}(t)$, satisfying the following equation:

$$
\begin{aligned}
u^{*}(t)= & \frac{1}{\Gamma(\epsilon)} \int_{t_{k-1}}^{t}(t-\tau)^{\epsilon-1} \phi_{q}\left(\mathcal{G}\left(u^{*}(\tau)\right)\right) d \tau+\frac{\sum_{k=1}^{m}}{\Gamma(\epsilon)} \int_{t_{k-1}}^{t_{k}}\left(t_{k}-\tau\right)^{\epsilon-1} \phi_{q}\left(\mathcal{G}\left(u^{*}(\tau)\right)\right) d \tau \\
& +\sum_{k=1}^{m} I_{k}\left(u^{*}\left(t_{k}\right)\right)
\end{aligned}
$$

with

$$
\left|u(t)-u^{*}(t)\right| \leq \mathcal{D}^{*} \lambda^{*}
$$

Theorem 3.2 With the help of assumptions $\left(\mathcal{A}_{1}\right)-\left(\mathcal{A}_{3}\right)$, the impulsive fractional order $D E$ (1.3) is Hyers-Ulam stable.

Proof For the Hyers-Ulam stability of the integral equation (1.3), with assumptions $\left(\mathcal{A}_{1}\right)$ $\left(\mathcal{A}_{3}\right)$ and Lemma 1.2, we have

$$
\begin{aligned}
\mid u(t) & -u^{*}(t) \mid \\
\leq & \frac{1}{\Gamma(\epsilon)} \int_{t_{k-1}}^{t}(t-\tau)^{\epsilon-1}\left|\phi_{q}(\mathcal{G}(u(\tau)))-\phi_{q}\left(\mathcal{G}\left(u^{*}(\tau)\right)\right)\right| d \tau \\
& +\frac{\sum_{k=1}^{m}}{\Gamma(\epsilon)} \int_{t_{k-1}}^{t_{k}}\left(t_{k}-\tau\right)^{\epsilon-1}\left|\phi_{q}(\mathcal{G}(u(\tau)))-\phi_{q}\left(\mathcal{G}\left(u^{*}(\tau)\right)\right)\right| d \tau \\
& +\sum_{k=1}^{m}\left|I_{k}\left(u\left(t_{k}\right)\right)-I_{k}\left(u^{*}\left(t_{k}\right)\right)\right|
\end{aligned}
$$




$$
\begin{aligned}
& \leq(q-1) \mathcal{M}^{q-2} \frac{1}{\Gamma(\epsilon)} \int_{t_{k-1}}^{t}(t-\tau)^{\epsilon-1} \\
& \times\left(\frac{1}{\Gamma(\gamma)} \int_{\tau_{1}}^{\tau}(\tau-s)^{\gamma-1}\left|\psi_{1}(s, u(s))-\psi_{1}\left(s, u^{*}(s)\right)\right| d s\right. \\
& +\frac{\sum_{k=1}^{m}}{\Gamma(\gamma)} \int_{\tau_{k-1}}^{\tau_{k}}\left(\tau_{k}-s\right)^{\gamma-1}\left|\psi_{1}(s, u(s))-\psi_{1}\left(s, u^{*}(s)\right)\right| d s \\
& +\sum_{k=1}^{m} \frac{\left(t-\tau_{k}\right)}{\Gamma(\gamma-1)} \int_{\tau_{k-1}}^{\tau_{k}}\left(t_{1}-s\right)^{\gamma-2}\left|\psi_{1}(s, u(s))-\psi_{1}\left(s, u^{*}(s)\right)\right| d s \\
& +\frac{\tau}{1-\lambda^{*}}\left[\frac{\lambda^{*}}{\Gamma(\gamma-1)} \int_{0}^{1}(1-s)^{\gamma-2}\left|\psi_{1}(s, u(s))-\psi_{1}\left(s, u^{*}(s)\right)\right| d s\right. \\
& +\frac{\sum_{k=1}^{m}\left(\lambda^{*}-1+\tau_{k}\right)}{\Gamma(\gamma-1)} \int_{\tau_{k-1}}^{\tau_{k}}\left(\tau_{k}-s\right)^{\gamma-2}\left|\psi_{1}(s, u(s))-\psi_{1}\left(s, u^{*}(s)\right)\right| d s \\
& +\frac{1}{\Gamma(\gamma)} \int_{0}^{1}(1-s)^{\gamma-1}\left|\psi_{1}(s, u(s))-\psi_{1}\left(s, u^{*}(s)\right)\right| d s \\
& \left.\left.+\frac{\sum_{k=}^{m}}{\Gamma(\gamma)} \int_{\tau_{k-1}}^{\tau_{k}}\left(\tau_{k}-s\right)^{\gamma-1}\left|\psi_{1}(s, u(s))-\psi_{1}\left(s, u^{*}(s)\right)\right| d s\right]\right) d \tau \\
& +(q-1) \mathcal{M}^{q-2} \frac{\sum_{k=1}^{m}}{\Gamma(\epsilon)} \int_{t_{k-1}}^{t_{k}}\left(t_{k}-\tau\right)^{\epsilon-1} \\
& \times\left(\frac{1}{\Gamma(\gamma)} \int_{\tau_{1}}^{\tau}(\tau-s)^{\gamma-1}\left|\psi_{1}(s, u(s))-\psi_{1}\left(s, u^{*}(s)\right)\right| d s\right. \\
& +\frac{\sum_{k=1}^{m}}{\Gamma(\gamma)} \int_{\tau_{k-1}}^{\tau_{k}}\left(\tau_{k}-s\right)^{\gamma-1}\left|\psi_{1}(s, u(s))-\psi_{1}\left(s, u^{*}(s)\right)\right| d s \\
& +\sum_{k=1}^{m} \frac{\left(\tau-\tau_{k}\right)}{\Gamma(\gamma-1)} \int_{\tau_{k-1}}^{\tau_{k}}\left(\tau_{1}-s\right)^{\gamma-2}\left|\psi_{1}(s, u(s))-\psi_{1}\left(s, u^{*}(s)\right)\right| d s \\
& +\frac{\tau}{1-\lambda^{*}}\left[\frac{\lambda^{*}}{\Gamma(\gamma-1)} \int_{0}^{1}(1-s)^{\gamma-2}\left|\psi_{1}(s, u(s))-\psi_{1}\left(s, u^{*}(s)\right)\right| d s\right. \\
& +\frac{\sum_{k=1}^{m}\left(\lambda^{*}-1+\tau_{k}\right)}{\Gamma(\gamma-1)} \int_{\tau_{k-1}}^{\tau_{k}}\left(\tau_{k}-s\right)^{\gamma-2}\left|\psi_{1}(s, u(s))-\psi_{1}\left(s, u^{*}(s)\right)\right| d s \\
& +\frac{1}{\Gamma(\gamma)} \int_{0}^{1}(1-s)^{\gamma-1}\left|\psi_{1}(s, u(s))-\psi_{1}\left(s, u^{*}(s)\right)\right| d s \\
& \left.\left.+\frac{\sum_{k=1}^{m}}{\Gamma(\gamma)} \int_{\tau_{k-1}}^{\tau_{k}}\left(\tau_{k}-s\right)^{\gamma-1}\left|\psi_{1}(s, u(s))-\psi_{1}\left(s, u^{*}(s)\right)\right| d s\right]\right) d \tau \\
& +\sum_{k=1}^{m}\left|I_{k}\left(u\left(t_{k}\right)\right)-I_{k}\left(u^{*}\left(t_{k}\right)\right)\right| \\
& \leq \frac{(q-1) \mathcal{M}^{q-2}}{\Gamma(\epsilon+1)}\left[\left(\frac{1+m}{\Gamma(\gamma+1)}+\frac{1}{1-\lambda^{*}}\left[\frac{\lambda^{*}(1+m)}{\Gamma(\gamma)}+\frac{1+m}{\Gamma(1+\gamma)}\right]\right) \mathcal{L}_{\psi_{1}}\left|u-u^{*}\right|\right] \\
& +\frac{m(q-1) \mathcal{M}^{q-2}}{\Gamma(\epsilon+1)}\left[\left(\frac{1+m}{\Gamma(1+\gamma)}+\frac{1}{1-\lambda^{*}}\left[\frac{\lambda^{*}(1+m)}{\Gamma(\gamma)}+\frac{1+m}{\Gamma(1+\gamma)}\right]\right) \mathcal{L}_{\psi_{1}}\left|u-u^{*}\right|\right] \\
& +m \mathcal{J}\left|u-u^{*}\right|
\end{aligned}
$$




$$
\begin{aligned}
\leq & {\left[(q-1) \mathcal{M}^{q-2} \frac{1+m}{\Gamma(1+\epsilon)}\left(\frac{1+m}{\Gamma(\gamma+1)}+\frac{1}{1-\lambda^{*}}\left[\frac{\lambda^{*}(1+m)}{\Gamma(\gamma)}+\frac{m+1}{\Gamma(1+\gamma)}\right]\right) \mathcal{L}_{\psi_{1}}\right.} \\
& +m \mathcal{J}]\left\|u-u^{*}\right\| .
\end{aligned}
$$

\section{Example/application}

For the application of the theorems proved in Sects. 3 and 4, the following example is presented.

Example 4.1 Assume that $\psi_{1}(t, u(t))=\frac{|u(t)|}{100+|u(t)|}$, for $t \in[0,1], p=3$. Consider the following impulsive fractional order DE as a particular example of (1.3):

$$
\left\{\begin{array}{l}
\mathcal{D}^{\gamma=1.5}\left[\phi_{p=3}\left[\mathcal{D}^{\epsilon=0.5} u(t)\right]\right]-\psi_{1}(t, u(t))=0, \\
\Delta\left[\phi_{p=3}\left[\mathcal{D}^{\epsilon=0.5} u\left(t_{k}\right)\right]\right]=\frac{1}{100}, \quad \Delta\left[\phi_{p=3}\left[\mathcal{D}^{\epsilon=0.5} u\left(t_{k}\right)\right]\right]^{\prime}=\frac{1}{100}, \\
\Delta u\left(t_{k}\right)=I_{k}\left(u\left(t_{k}\right)\right), \\
\phi_{p}\left(\mathcal{D}^{\epsilon=0.5} u(0)\right)=0, \quad \phi_{p}\left(\mathcal{D}^{\epsilon=0.5} u(1)\right)=\lambda^{*} \phi_{p}^{\prime}\left(\mathcal{D}^{\epsilon=0.5} u(1)\right), \quad u(0)=0 .
\end{array}\right.
$$

By a simple calculation, we have the values $\lambda^{*}=\mathcal{M}_{0}=\mathcal{L}_{\psi_{1}}=b_{k}=c_{k}=\frac{1}{100}, m=30, k=$ $1,2, \ldots, 30$. The conditions $\left(\mathcal{R}_{1}\right),\left(\mathcal{R}_{2}\right)$ are satisfied and $\Delta^{*}<1$ for

$$
\begin{aligned}
\Delta^{*}= & {\left[(q-1) \mathcal{M}^{q-2} \frac{m+1}{\Gamma(\epsilon+1)}\left(\frac{1+m}{\Gamma(\gamma+1)}+\frac{1}{1-\lambda^{*}}\left[\frac{\lambda^{*}(1+m)}{\Gamma(\gamma)}+\frac{m+1}{\Gamma(1+\gamma)}\right]\right) \mathcal{L}_{\psi_{1}}\right.} \\
& +m \mathcal{J}] .
\end{aligned}
$$

Thus, with the help of Theorem 2.4, the impulsive fractional order DE (4.1), has a unique solution and is Hyers-Ulam stable.

\section{Conclusion}

In this paper, we have considered a generalized class of impulsive fractional order DEs for the study of EUS and Hyers-Ulam stability. For these goals of the paper, we converted the suggested FODE with impulsive effect and nonlinear $\phi_{p}$ operator to an equivalent fractional order integral system involving $\phi_{q}=\phi_{p}^{-1}$. Then, with the help of classical fixed point theorems, the existence results are obtained and stability is examined. For application, an illustrative example is given. For future work, we suggest to consider the problem with singularity and delay for different fractional order derivatives.

\section{Acknowledgements}

All the authors are thankful to the editorial board and anonymous reviewers whose comments improved the quality of the paper. The third author (Thabet Abdeljawad) would like to thank Prince Sultan University for funding this work through research group in Applied Mathematics (NAMAM) group number RG-DES-201701-17.

Funding

Prince Sultan University for funding this work through research group in Applied Mathematics (NAMAM) group number RG-DES-201701-17.

Competing interests

The authors have no conflict of interests regarding the publication of this paper. 


\section{Author details}

${ }^{1}$ College of Engineering Mechanics and Materials, Hohai University, Nanjing, P.R. China. ${ }^{2}$ Department of Mathematics, Shaheed BB University, Sheringal, Pakistan. ${ }^{3}$ Department of Mathematics, University of Peshawar, Peshawar, Pakistan. ${ }^{4}$ Department of Mathematics and General Sciences, Prince Sultan University, Riyadh, Saudi Arabia. ${ }^{5}$ College of Science, Hohai University, Nanjing, P.R. China.

\section{Publisher's Note}

Springer Nature remains neutral with regard to jurisdictional claims in published maps and institutional affiliations.

Received: 1 October 2018 Accepted: 15 January 2019 Published online: 22 January 2019

\section{References}

1. Samko, S.G., Kilbas, A.A., Marichev, O.I.: Fractional Integrals and Derivatives: Theory and Applications. Gordon \& Breach, Yverdon (1993)

2. Podlubny, I.: Fractional Differential Equations. Academic Press, New York (1999)

3. Hilfer, R. (ed.): Application of Fractional Calculus in Physics. World Scientific, Singapore (2000)

4. Kilbas, A.A., Srivastava, H.M., Trujillo, J.J.: Theory and Applications of Fractional Differential Equations. North-Holland Mathematics Studies, vol. 24. Elsevier, Amsterdam (2006)

5. Deimling, K.: Nonlinear Functional Analysis. Springer, New York (1985)

6. Bolandtalat, A., Babolian, E., Jafari, H.: Numerical solutions of multi-order fractional differential equations by Boubaker polynomials. Open Phys. 14, 226-230 (2016)

7. Jafari, H., Jassim, H.K., Moshokoa, S.P., Ariyan, V.M., Tchier, F.: Reduced differential transform method for partial differential equations within local fractional derivative operators. Adv. Mech. Eng. 8(4), 1-6 (2016)

8. Anastassiou, G.A.: On right fractional calculus. Chaos Solitons Fractals 42(1), 365-376 (2009)

9. Agarwal, R., Hristova, S., O'Regan, D.: A survey of Lyapunov functions, stability and impulsive Caputo fractional differential equations. Fract. Calc. Appl. Anal. 19(2), 290-318 (2016)

10. Wang, J., Fečkan, M., Zhou, Y.: A survey on impulsive fractional differential equations. Fract. Calc. Appl. Anal. 19(4)، 806-831 (2016)

11. Hu, Z., Liu, W., Liu, J.: Existence of solutions for a coupled system of fractional $p$-Laplacian equations at resonance. Adv. Differ. Equ. 2013, 312 (2013)

12. Jafari, H., Baleanu, D., Khan, H., Khan, R.A., Khan, A.: Existence criterion for the solutions of fractional order p-Laplacian boundary value problems. Bound. Value Probl. 2015, 164 (2015). https://doi.org/10.1186/s13661-015-0425-2

13. Khan, H., Chen, W., Sun, H.: Analysis of positive solution and Hyers-Ulam stability for a class of singular fractional differential equations with p-Laplacian in Banach space. Math. Methods Appl. Sci. 41(9), 3430-3440 (2018)

14. Baleanu, D., Khan, H., Jafari, H., Khan, R.A., Alipour, M.: On existence results for solutions of a coupled system of hybrid boundary value problems with hybrid conditions. Adv. Differ. Equ. 2015, 31 (2015)

15. Khan, A., Seyam, M.I., Zada, A., Khan, H.: Stability analysis of nonlinear fractional differential equations with Caputo and Riemann-Liouville derivatives. Eur. Phys. J. Plus 133, 264 (2018). https://doi.org/10.1140/epjp/i2018-12119-6

16. Raheem, A., Maqbul, M.D.: Oscillation criteria for impulsive partial fractional differential equations. Comput. Math. Appl. 73(8), 1781-1788 (2017)

17. Wang, J., Zhang, Y.: On the concept and existence of solutions for fractional impulsive systems with Hadamard derivatives. Appl. Math. Lett. 39, 85-90 (2015)

18. Cong, N.D., Tuan, H.T.: Existence, uniqueness, and exponential boundedness of global solutions to delay fractional differential equations. Mediterr. J. Math. 14, 193 (2017). https://doi.org/10.1007/s00009-017-0997-4

19. Abaas, A., Benchohra, M., Alsaedi, A., Zhou, Y.: Stability results for partial fractional differential equations with noninstantaneous impulses. Adv. Differ. Equ. 2017, 75 (2017). https://doi.org/10.1186/s13662-017-1110-9

20. Liu, Z., Lu, L., Szanto, I.: Existence of solutions for fractional impulsive differential equations with $p$-Laplacian operator. Acta Math. Hung. 141(3), 203-219 (2013). https://doi.org/10.1007/s10474-013-0305-0

21. Feckan, M., Zhou, Y., Wang, J.: On the concept and existence of solution for impulsive fractional differential equations Commun. Nonlinear Sci. Numer. Simul. 17, 3050-3060 (2012)

22. Baleanu, D., Agarwal, R.P., Khan, H., Khan, R.A., Jafari, H.: On the existence of solution for fractional differential equations of order $3<\delta \leq 4$. Adv. Differ. Equ. 2015, 362 (2015)

23. Zada, A., Ali, W., Farina, S.: Hyers-Ulam stability of nonlinear differential equations with fractional integrable impulses. Math. Methods Appl. Sci. 40(15), 5502-5514 (2017). https://doi.org/10.1002/mma.4405

24. Isaia, F.: On a nonlinear integral equation without compactness. Acta Math. Univ. Comen. LXXV(2), $233-240$ (2006)

25. Urs, C.: Coupled fixed point theorems and applications to periodic boundary value problems. Miskolc Math. Notes 14(1), 323-333 (2013)

26. Khan, A., Li, Y., Shah, K., Khan, T.S.: On coupled $p$-Laplacian fractional differential equations with nonlinear boundary conditions. Complexity 2017, Article ID 8197610 (2017)

27. Khan, H., Tunc, C., Chen, W., Khan, A.: Existence theorems and Hyers-Ulam stability for a class of hybrid fractional differential equations with $p$-Laplacian operator. J. Appl. Anal. Comput. 8(4), 1211-1226 (2018) 